\title{
PAPER
}

\section{Using the SF-36 measure to compare the health impact of multiple sclerosis and Parkinson's disease with normal population health profiles}

\author{
A Riazi, J C Hobart, D L Lamping, R Fitzpatrick, J A Freeman, C Jenkinson, V Peto, \\ A J Thompson
}

See end of article for authors' affiliations

.......................

Correspondence to: Dr Jeremy Hobart, Department of Clinical Neurosciences, Derriford Hospital, Plymouth, Devon PL8 8DH, UK jeremy.hobart@ phnt.swest.nhs.uk

Received 17 April 2002 In final revised form

10 January 2003

Accepted

15 January 2003

\begin{abstract}
Objective: To examine the relative impact of two chronic neurological disorders, multiple sclerosis and Parkinson's disease, by comparing patients' scores on the medical outcomes study 36-item short form health survey (SF-36) with the health profile of the United Kingdom population norms.

Methods: 638 people representing the full spectrum of multiple sclerosis and 227 patients with Parkinson's disease were studied. Health status was measured by the SF-36. Scores for the eight health domains were compared after controlling for age, sex, disease duration, mobility, social class, ethnicity, education, marital status, and employment status.

Results: People with multiple sclerosis and those with Parkinson's disease had significantly worse health than the general population on all eight domains measured by the SF-36. The relative impact of multiple sclerosis and Parkinson's disease were similar, but multiple sclerosis resulted in poorer scores on physical functioning and better scores in mental health. People with mild multiple sclerosis who walked without an aid also had significantly worse scores in all dimensions than the general UK population.

Conclusions: The results highlight the need for further research into aspects of health measured by the SF-36. Nevertheless, generic measures that are applicable across multiple diseases may fail to address clinically important aspects of the impact of specific disorders.
\end{abstract}

$\mathrm{t}$ is increasingly recognised that health care should be evidence based, and that health care evaluations should incorporate the patient's perspective. This has led to the development and use of patient based health status rating scales as outcome measures for clinical trials, audit, and epidemiological studies. The medical outcomes study 36-item short form health survey (SF-36) ${ }^{1}$ is the most widely used patient based generic measure of health. It generates a profile of scores for eight health dimensions.

Patient reported health status data from group studies are valuable as they reflect the impact of disease from the patient's perspective. Thus the health profiles of different conditions can be compared to estimate this relative burden. ${ }^{1}$ Information from such measures could identify areas where patients are experiencing particular problems, where further investigation or research is needed, and where clinicians may be able to offer help. This might eventually mean that services that meet patients' needs can be developed on a stronger evidence base.

Several studies have examined health status in multiple sclerosis $^{2-7}$ and Parkinson's disease. ${ }^{8-12}$ However, as far as we are aware none has controlled for sociodemographic variables other than age and sex when making comparisons between the two disorders or with controls, and most have been in small samples from a limited population.

This study builds on previous work by examining the impact of multiple sclerosis and Parkinson's disease on the eight health dimensions of the SF-36, in samples of patients from across the United Kingdom. The health profiles of patients with multiple sclerosis and Parkinson's disease were compared with each other and with UK norms, controlling for sociodemographic variables. Our aim was to examine the relative impact of multiple sclerosis and Parkinson's disease by comparing them with the health profile of UK norms.

\section{METHODS}

Three groups were studied: patients with multiple sclerosis, patients with Parkinson's disease, and United Kingdom norms as a reference group.

\section{Multiple sclerosis}

Patients were derived from three separate sources.

Sample 1 was a postal survey of 500 randomly selected and geographically stratified members of the Multiple Sclerosis Society of Great Britain and Northern Ireland. This was part of a larger study developing a patient based outcome measure for multiple sclerosis. ${ }^{13}$ The SF-36 was administered in a booklet along with three other health measures and demographic questions. Non-responders were sent reminders at three and five weeks.

Sample 2 consisted of adults with clinically definite multiple sclerosis attending the National Hospital for Neurology and Neurosurgery in London. Full details of the sampling and the stratification process for this sample are described elsewhere. ${ }^{14}$ Briefly, 150 consecutive attenders were recruited from a weekly outpatient clinic, from an inpatient neurological rehabilitation unit, and from admissions under a single consultant (AJT).

Sample 3 consisted of adults with clinically definite multiple sclerosis participating in a study examining the responsiveness of a patient based outcome measure for people with multiple sclerosis. This sample consisted of three subsamples: patients who were consecutively admitted to the National Hospital for Neurology and Neurosurgery between February and November 2000 for rehabilitation (subsample 1) or intravenous steroid treatment of relapses (subsample 2); these two subsamples completed the SF-36 along with other health measures and demographic questions at time 1 (admission) and time 2 (discharge for rehabilitation group; six weeks later for the steroid group). In addition, the questionnaires were 


\begin{tabular}{|c|c|c|c|}
\hline Characteristic & MS (n (\%)) & PD (n (\%)) & UK norms (n (\%)) \\
\hline Total number of subjects & 638 & 227 & 2056 \\
\hline \multicolumn{4}{|l|}{ Sex } \\
\hline Female & $412(65)$ & $92(40)$ & $1122(55)$ \\
\hline Male & $219(35)$ & $135(60)$ & $929(45)$ \\
\hline \multicolumn{4}{|l|}{ Age range } \\
\hline $20-39$ years & $146(24)$ & $0(0)$ & 705 (37) \\
\hline $40-59$ years & $357(57)$ & $26(13)$ & $612(30)$ \\
\hline$>60$ years & 118 (19) & $196(87)$ & 644 (33) \\
\hline \multicolumn{4}{|l|}{ Time since diagnosis } \\
\hline 0-9 years & $309(51)$ & $137(63)$ & $\mathrm{n} / \mathrm{a}$ \\
\hline 10-19 years & $200(33)$ & $59(27)$ & $\mathrm{n} / \mathrm{a}$ \\
\hline$>20$ years & $100(16)$ & $23(10)$ & $\mathrm{n} / \mathrm{a}$ \\
\hline \multicolumn{4}{|l|}{ Ethnicity* } \\
\hline White & $464(96)$ & $\mathrm{n} / \mathrm{a}$ & 1981 (97) \\
\hline Others & $21(4)$ & $\mathrm{n} / \mathrm{a}$ & $68(3)$ \\
\hline \multicolumn{4}{|l|}{ Marital status } \\
\hline Married or with partner & 447 (71) & $169(75)$ & $1224(60)$ \\
\hline No & $186(29)$ & $55(25)$ & $831(40)$ \\
\hline \multicolumn{4}{|l|}{ Employment status } \\
\hline Working & $144(24)$ & $2(1)$ & $1001(50)$ \\
\hline No & $482(76)$ & 222 (99) & $994(50)$ \\
\hline \multicolumn{4}{|l|}{ Education* } \\
\hline Obtained degree or professional qualification & $155(33)$ & $\mathrm{n} / \mathrm{a}$ & $\mathrm{n} / \mathrm{a}$ \\
\hline No & $321(67)$ & $\mathrm{n} / \mathrm{a}$ & $\mathrm{n} / \mathrm{a}$ \\
\hline \multicolumn{4}{|l|}{ Social class* } \\
\hline Manual social class & $185(41)$ & $90(50)$ & $940(48)$ \\
\hline No & $267(59)$ & $91(50)$ & $1032(52)$ \\
\hline \multicolumn{4}{|l|}{ Mobility indoors* } \\
\hline Walk unaided & $136(29)$ & $\mathrm{n} / \mathrm{a}$ & $\mathrm{n} / \mathrm{a}$ \\
\hline Walk with an aid & $204(43)$ & $\mathrm{n} / \mathrm{a}$ & $\mathrm{n} / \mathrm{a}$ \\
\hline Uses wheelchair & $130(28)$ & $\mathrm{n} / \mathrm{a}$ & $\mathrm{n} / \mathrm{a}$ \\
\hline
\end{tabular}

posted nine months apart to people with a confirmed diagnosis of primary progressive multiple sclerosis ${ }^{15}$ from a clinical database (subsample $3 ; \mathrm{n}=119$ ). Non-responders were sent reminders at three and five weeks. Only the SF-36 scores at time 1 are used in the present analyses.

In the rehabilitation and steroid subsamples, subjects were excluded if they appeared to have cognitive impairment (substantiated by neuropsychological testing) which precluded reliable completion of questionnaires, if they had other comorbid disabling disorders, or if they were not English speaking.

\section{Parkinson's disease}

Data for patients with Parkinson's disease $(n=227)$ were obtained from a population survey of Parkinson's Disease Society members. Full details of the sampling and the stratification process for this sample are described elsewhere. ${ }^{10}$

Briefly, the sample was identified from membership of local branches of the Parkinson's Disease Society from five areas of England. Questionnaires were sent to 405 individuals. No reminders were sent.

\section{United Kingdom norms}

The UK population data $(n=2056)$ were obtained from the Office of National Statistics Omnibus Survey, archived by the University of Essex. ${ }^{16}$

\section{Health status assessment}

The SF-36 is a widely used generic measure of health status. Thirty five of the 36 items are grouped into eight scales that address health constructs considered to be important to most health care situations: physical functioning, role limitations (physical problems), bodily pain, general health, vitality, social functioning, role limitations (emotional problems), and mental health. One item assesses perception of changes in health but is not used to compute scale scores. Methods for comput- ing scores, which range from 0 to 100 , are reported elsewhere.

\section{Statistical analyses}

When comparing scores of different samples it is important to control for variables that might influence the results. Multiple linear regression analysis is a method for investigating the extent to which one or more predictive variables (independent variables) predict an outcome variable (dependent variable). It is often used to identify whether some critical variable adds additional predictive value to the predictive equation for a dependent variable after other independent variables have already entered the equation. ${ }^{18}$

We entered the grouping variable (multiple sclerosis $v$ general population, Parkinson's disease $v$ general population, or multiple sclerosis $v$ Parkinson's disease) and the sociodemographic variables in a separate regression equation to predict each of the SF-36 dimensions. This determined whether there were significant differences in SF-36 scores between the groups after controlling for the sociodemographic variables.

In the comparison of multiple sclerosis patients and the general population norms, the regression analyses controlled for social class, ethnicity, education level, sex, marital status, employment status, and age. Within the multiple sclerosis sample, subgroup analyses were conducted according to patients' level of mobility indoors (walking without aid, walking with support, using wheelchair). Multiple linear regression analyses adjusting for age, sex, marital status, social class, employment, ethnicity, and duration of multiple sclerosis were used to establish differences in SF-36 scores between subgroups, and between each of the subgroups and the general population norms.

In the comparison of Parkinson's disease patients and general population norms, age, sex, marital status, social class, and 
employment status were controlled. In the comparison of multiple sclerosis and Parkinson's disease patients, age, sex, marital status, social class, employment, and years since diagnosis of multiple sclerosis or Parkinson's disease were controlled.

\section{RESULTS}

\section{Sample characteristics}

Multiple sclerosis-Completed questionnaires were obtained from 638 people (sample 1, 288; sample 2, 149; sample 3, 201). In sample 1 the response rate was $69 \%$; in sample 2, it was $99 \%$ after one person withdrew; in sample 3, four people in the rehabilitation subsample were excluded because of cognitive impairment; in the primary progressive cohort, 104 questionnaires were returned, a response rate of $87 \%$.

Parkinson's disease-Eleven people were subsequently removed from the denominator as they were deceased or did not have Parkinson's disease. In all, 227 questionnaires were returned, a response rate of $57.6 \%$.

The characteristics of the three groups are shown in table 1. In the multiple sclerosis sample, data for ethnicity, education, social class, and mobility were available from 489 people as they were not collected in sample 2. In the Parkinson's disease sample, ethnicity, education, and mobility data were not collected.

There are some expected differences between the three samples. The multiple sclerosis sample was predominantly female. The majority of the Parkinson's disease sample was aged 60 or over. Although the general UK population included an equal proportion of those who were employed and unemployed, three quarters of the multiple sclerosis sample and 99\% of the Parkinson's disease sample were not employed. There were other similarities and differences worthy of note. The UK norms were younger than the Parkinson's disease or multiple sclerosis patients and consisted of fewer married individuals. The lower response rate for the Parkinson's disease patients may have been a result of the absence of reminders.

\section{Group comparison of SF-36 data}

Multiple sclerosis $v$ the general population

Participants with multiple sclerosis had lower mean scores on all dimensions of the SF-36 compared with the UK norms after controlling for sociodemographic variables $(p<0.001)$ (fig 1). The differences in scores were larger for the two physical domains of the SF-36 profile: physical function ( 58 points) and role limitations-physical (56 points); substantial for five domains: social function (35 points), role limitationsemotional ( 31 points), general health ( 25 points), vitality (25 points), and bodily pain ( 21 points); and small for the mental health dimension (10 points) (fig 1). This indicates that relative to the UK norms, multiple sclerosis has the greatest impact on the physical function and role limitations-physical dimensions. All differences were significant at $\mathrm{p}<0.001$.

\section{Multiple sclerosis subgroups defined by level of indoor mobility}

Figure 2 compares SF-36 data for three groups defined by mobility indoors. Less physically disabled individuals had significantly higher scores $(\mathrm{p}<0.05)$ on all SF-36 dimensions than those who used support when walking. Compared with those who used a wheelchair, those who walked independently had better scores $(p<0.05)$ in all dimensions except role limitations-emotional and mental health dimensions, where the difference was minimal (differences of 6 points and 5 points, respectively).

A stepwise decrease in scores with disease progression was seen in the physical function scores. There was a ninefold decrease in physical function scores between those who walked independently and those who used a wheelchair (fig 2). The significant floor effect in those who walked with an aid

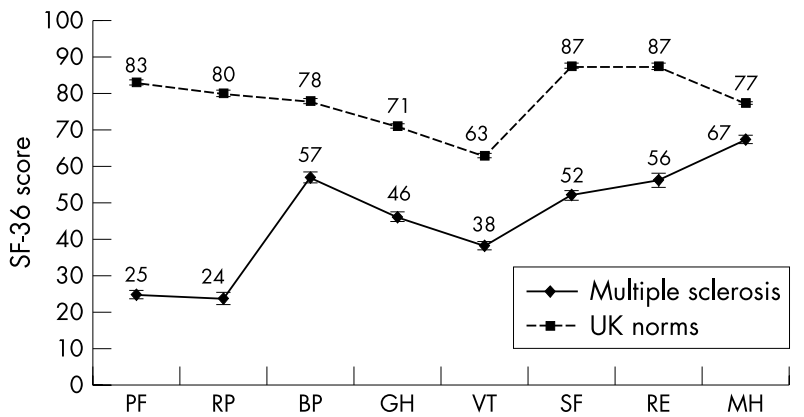

Figure 1 Graph showing SF-36 scores for multiple sclerosis patients compared with UK norms controlling for age, sex, marital status, social class, employment, and ethnicity. Error bars = SEM. BP bodily pain; $\mathrm{GH}$, general health perceptions; $M H$, mental health; PF physical functioning; RE, role emotional; RP, role physical; SF, social functioning; VT, vitality.

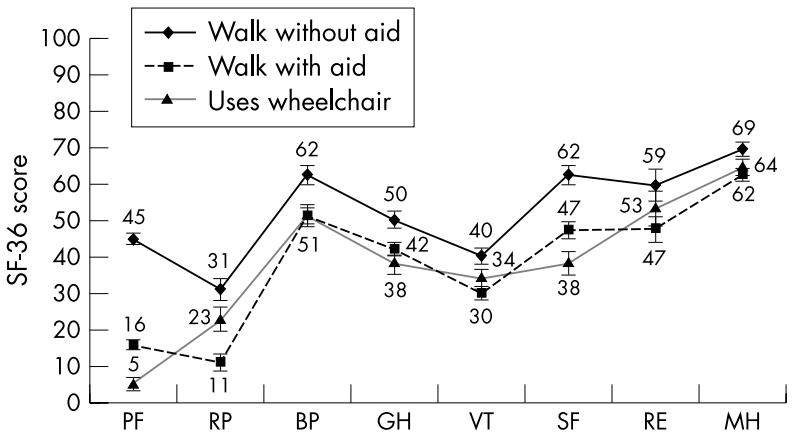

Figure 2 Graph showing SF-36 scores for the subgroups of multiple sclerosis patients controlling for age, sex, marital status, social class, employment, ethnicity, and years since diagnosis of multiple sclerosis. Error bars = SEM. BP, bodily pain; $\mathrm{GH}$, general health perceptions; $M H$, mental health; $\mathrm{PF}$, physical functioning; $\mathrm{RE}$, role emotional; RP, role physical; SF, social functioning; VT, vitality.

(14.2\%) and those who used wheelchairs $(67.8 \%)$ may have led to an underestimation of the differences between these two groups. A lesser stepwise decrease with disease progression was seen in social function and general health scores. Stepwise decreases in scores were not seen in the bodily pain, vitality, role limitations-emotional, mental health, and role limitations-physical dimensions. Those who walked with support scored markedly worse in the role limitationsphysical dimension, with the difference to the "walk without aid" group being approximately threefold. The three subgroups scored similarly on the mental health dimension (69, 62 , and 64 points for walk without aid, walk with aid, and wheelchair groups, respectively).

The SF-36 data for each severity group were compared with those of the general population (data not shown) and even the least physically disabled individuals showed a significant decrease in all dimension scores $(p<0.001)$. This difference was greatest for the role limitations-physical (difference of 45 points) and least for the mental health dimension (difference of 6 points). People who walked with an aid had significantly lower scores in all dimensions compared with the general population scores $(p<0.001)$, and in particular, the role limitations-physical dimension scores were 66 points lower than the normal population scores. Wheelchair users also had lower scores in all dimensions compared with the general population scores $(p<0.001)$ and as expected, markedly lower scores in the physical function dimension (difference of 75 points). Again, the least difference was for the mental health domain (difference of 9 points). 


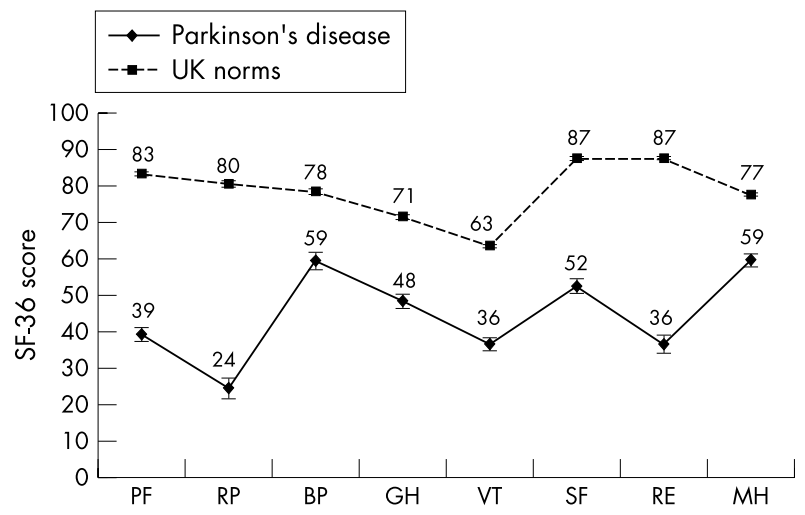

Figure 3 Graph showing SF-36 scores for patients with Parkinson's disease compared with UK norms controlling for age, sex, marital status, social class, and employment. Error bars = SEM. BP, bodily pain; $\mathrm{GH}$, general health perceptions; $M H$, mental health; PF, physical functioning; RE, role emotional; RP, role physical; SF, social functioning; VT, vitality.

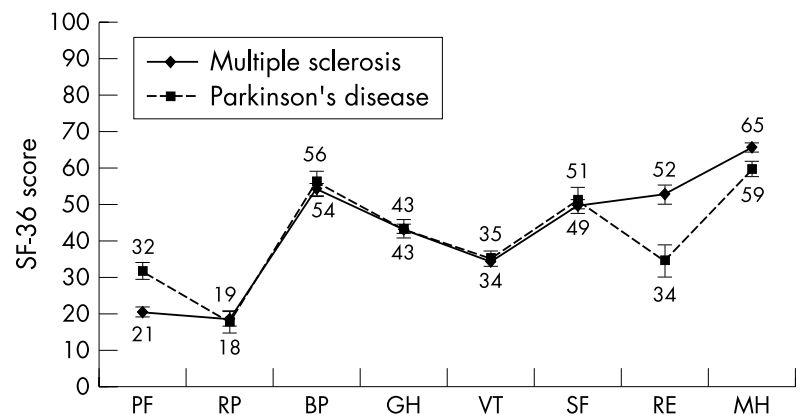

Figure 4 Graph showing SF-36 scores for multiple sclerosis patients compared with Parkinson's disease patients, controlling for age, sex, marital status, social class, employment, and years since diagnosis of multiple sclerosis or Parkinson's disease. Error bars $=$ SEM. BP, bodily pain; GH, general health perceptions; $\mathrm{MH}$, mental health; PF, physical functioning; RE, role emotional; RP, role physical; SF, social functioning; VT, vitality.

\section{Parkinson's disease $v$ the general population}

Participants with Parkinson's disease also had significantly lower mean scores for all health dimensions of the SF-36 compared with the UK norms controlling for sociodemographic variables $(p<0.001)$ (fig 3 ). The differences in profiles were very large for two dimensions: role limitationsphysical (56 points) and role limitations-emotional (51 points); and substantial for six dimensions: physical function (44 points), social function (35 points), vitality ( 27 points), general health (23 points), bodily pain (19 points), and mental health (18 points).

\section{Multiple sclerosis $v$ Parkinson's disease}

The relative impact of multiple sclerosis and Parkinson's disease were similar after controlling for duration of illness and other sociodemographic variables (fig 4). The two disease groups had similar profiles for role limitations-physical (19 and 18 , respectively), bodily pain ( 54 and 56 ), general health (43 and 43), vitality ( 34 and 35), and social function (49 and 51) ( $p>0.05$ for all comparisons). However, multiple sclerosis patients had lower mean scores for physical function (difference 11 points; $p<0.001$ ) but higher scores for the mental health dimension (difference 6 points; $p<0.05$ ). The greatest difference between the two groups was in the role limitations-emotional dimension, where the multiple sclerosis group scored substantially better than the Parkinson's disease group (difference 18 points; $\mathrm{p}<0.005$ ).

\section{DISCUSSION}

In this study we compared the health profile of a large sample of patients with multiple sclerosis, a smaller sample of patients with Parkinson's disease, and the UK norms, controlling for multiple sociodemographic variables. In the comparison of multiple sclerosis with Parkinson's disease, disease duration was also controlled. Results indicate that individuals with multiple sclerosis and Parkinson's disease have significantly worse health than the general population in all eight health domains measured by the SF-36. The differences in profiles of people with multiple sclerosis and the UK norms were largest in the physical function domain, and smallest in the mental health domain. The differences in profiles of people with Parkinson's disease and the UK norms were largest in the role limitations-physical and smallest in the mental health and bodily pain domains. Patients with multiple sclerosis and Parkinson's disease had similar health profiles in six of the eight dimensions, but multiple sclerosis resulted in poorer scores on the physical function and better scores in the mental health domain.

Subgroup analyses showed consistent health profiles across groups, but also provided some useful insights into the impact of multiple sclerosis. Even people who walk without an aid, and therefore might be viewed as having milder disease, had significantly worse scores in all dimensions compared with the general UK population. But the dramatic stepwise decrease in physical function scores as a result of increasing disability was not accompanied by a stepwise decrease in other health dimensions. Indeed, the differences in the subgroup profiles tended to be smallest for the mental health dimension. The most impaired group in the role limitationsphysical dimension was comprised of patients who required support for walking. Wheelchair users had better scores in role limitations-physical, vitality, role limitations-emotional, and mental health than people who needed an aid for walking.

In multiple sclerosis, the results from this UK sample support the findings of studies from Canada ${ }^{3}$ and Norway. ${ }^{2}$ The Norwegian study also found that the relative impact of multiple sclerosis was least for the mental health dimension. The Canadian study showed that significant impacts in all SF-36 dimensions were seen in patients with low expanded disability status scores (less physical disability), and those with less impairment of ambulation. Our results build on these studies. A large sample of multiple sclerosis patients was recruited from diverse clinical settings (community, inpatient rehabilitation, and inpatient steroids for multiple sclerosis relapses) and we controlled for multiple sociodemographic variables. These factors improve the general applicability of the results.

In Parkinson's disease, the results support the findings of two other studies in the United Kingdom. ${ }^{8}{ }^{10}$ Schrag et al used a sample of Parkinson's disease patients from a population based prevalence study and compared it with published quality of life (QoL) norms for the UK population. ${ }^{8}$ They found worse QoL scores in all areas except pain. In contrast, we showed a significant impact on pain. In the current study, after controlling for multiple sociodemographic variables in our comparisons with the general population scores, individuals with Parkinson's disease had poorer scores on all dimensions of QoL. Finally, as far as we are aware this is the first study to show similarities and differences between multiple sclerosis and Parkinson's disease, two chronic progressive disabling neurological disorders, by systematically controlling for potential confounding variables. However, in contrast to the multiple sclerosis patients, who were recruited from diverse settings, the Parkinson's disease sample was recruited solely from the community. This, and the low response rate in that sample, suggests that the sample may not have been fully representative-a specific weakness of our study.

The relatively similar mental health scores (compared with scores for other dimensions) between multiple sclerosis 
patients and the general UK population contrasts with published reports and clinical experience. Further analyses (available from the authors) showed that this result is consistent even when steroid treated patients are excluded from the analyses. Clinical experience suggests that multiple sclerosis is associated with substantial psychological distress. ${ }^{19}{ }^{20}$ One interpretation of our finding is that in our study, people with multiple sclerosis had adapted to the psychological demands of the illness. Another interpretation is that the SF-36 mental health dimension has limited validity as a measure of mental health in multiple sclerosis. Evidence in support of the latter interpretation comes from the finding that the SF-36 mental summary score (which incorporates the mental health scale) underestimates the impact of multiple sclerosis on mental health. ${ }^{21}$ Furthermore, the correlation between the SF-36 mental health and the psychological impact scale of the multiple sclerosis impact scale (MSIS-29), a disease specific measure developed from people with multiple sclerosis, is $0.76{ }^{13}$ This indicates that the constructs measured by these two scales are related but distinct, and far from identical (they have less than $60 \%$ shared variance). This finding highlights the fundamental importance of reliable and valid health measurements for evidence based medicine.

It is important to note that the SF-36 is not a needs assessment tool. It simply highlights areas of health impact that require further investigation for specific management. The results from our study support the need for further research (including the assessment of health care needs) that is sensitive to at least the eight constructs measured by the SF-36. In multiple sclerosis in particular, results suggest that this should include patients in the early stage of the disease. Further research could identify how patients cope, what their unmet needs are, and how clinicians might meet these needs (if that is indeed possible) and what services might be developed to support them in doing so.

\section{Conclusions}

Measures of health status, such as the SF-36, can detect areas of disease impact that may not be obvious from the clinical situation. This information, supplemented by further investigations such as the assessment of health care needs, is capable of guiding subsequent disease intervention. However, it is important to recognise that generic measures may fail to address clinically important aspects of the impact of a specific disease, and cross sectional studies do not define change in disease impact over time. Studies are required to determine the specific domains of health affected by diseases, as well as the natural history of the impact of neurological disorders on health status.

\section{ACKNOWLEDGEMENTS}

We wish to thank the people with multiple sclerosis and Parkinson's disease who freely participated in the studies that provided the data used here, along with the Multiple Sclerosis Society of Great Britain and Northern Ireland and the Parkinson's Society of the United Kingdom for their collaboration. This study was funded by a grant from the NHS health technology assessment programme, but the views and opinions expressed do not necessarily reflect those of the NHS executive. Material from Omnibus Survey is crown copyright; has been made available by the Office for National Statistics (ONS) through the UK data archive (UKDA) and has been used by permission. Neither the ONS nor the UKDA bear and responsibility for the analysis or interpretation of the data reported here. We are particularly grateful to one referee for helpful remarks and suggestions.

AR is generously supported by the de Laszlo Foundation.

\section{Authors' affiliations}

A Riazi, J C Hobart, J A Freeman, A J Thompson, Neurological Outcome Measures Unit, Institute of Neurology, Queen Square, London WCl, UK

D L Lamping, Health Services Research Unit, London School of Hygiene and Tropical Medicine, Keppel St, London WCI, UK

R Fitzpatrick, C Jenkinson, V Peto, Department of Public Health and

Primary Care, University of Oxford, Oxford, UK

Competing interests: none declared

\section{REFERENCES}

1 Ware JE, Snow KK, Kosinski M, et al. SF-36 health survey manual and interpretation guide. Boston, Massachusetts: Nimrod Press, 1993.

2 Nortvedt MW, Riise T, Myhr KM, et al. Quality of life in multiple sclerosis - measuring the disease effects more broadly. Neurology 1999;53:1098-103.

3 Canadian Burden of Illness Study Group. Burden of illness of multiple sclerosis: part II. Quality of life. Can J Neurol Sci 1998;25:31-8.

4 Ford HL, Gerry E, Johnson MH, et al. Health status and quality of life of people with multiple sclerosis. Disabil Rehabil 2001;23:516-21.

5 Herman BP, Vickery B, Hays RD, et al. A comparison of health-related quality of life in patients with epilepsy, diabetes and multiple sclerosis. Epilepsy Res 1996;25:113-18.

6 Rothwell PM, McDowell Z, Wong CK, et al. Doctors and patients don't agree: cross sectional study of patients' and doctors' perceptions and assessments of disability in multiple sclerosis. BM 1997;314:1580-3.

7 Sprangers MAG, de Regt EB, Andries F, et al. Which chronic conditions are associated with better or poorer quality of life? J Clin Epidemiol 2000:53:895-907.

8 Schrag A, Jahanshahi M, Quinn N. How does Parkinson's disease affect quality of life? A comparison with quality of life in the general population. Mov Disord 2000;15:1112-18.

9 Kuopio A, Marttila, R, Helenius $\mathrm{H}$, et al. The quality of life in Parkinson's disease. Mov Disord 2000;15:216-23.

10 Jenkinson C, Peto V, Fitzpatrick R, et al. Self-reported functioning and well-being in patients with Parkinson's disease - comparison of the short-form health survey (SF-36) and the Parkinson's disease questionnaire (PDQ-39). Age Ageing 1995;24:505-9.

11 Chrischilles EA, Rubenstein LM, Voelker MD, et al. The health burdens of Parkinson's disease. Mov Disord 1998;13:406-13.

12 Hobson P, Meara J. Self-reported functioning and well-being in patients with Parkinson's disease: comparison of the Short-Form 36 and the Parkinson's disease questionnaire. Age Ageing 1996;25:334-5.

13 Hobart JC, Lamping DL, Fitzpatrick R, et al. The multiple sclerosis impact scale (MSIS-29) - a new patient-based outcome measure. Brain $2001 ; 124: 962-73$

14 Freeman JA, Hobart JC, Langdon DW, et al. Clinical appropriateness: a key factor in outcome measure selection. The 36-item short form health survey in multiple sclerosis. J Neurol Neurosurg Psychiatry 2000; 68:150-6.

15 Thompson AJ, Montalban X, Barkhof F, et al. Diagnostic criteria for primary progressive multiple sclerosis: a position paper. Ann Neurology 2000;47:831-5.

16 OPCS. Omnibus survey. Colchester: The Data Archive, 1997.

17 Bowling A, Bond M, Jenkinson C, et al. Short Form 36 (SF-36) Health Survey questionnaire: which normative data should be used? Comparisons between the norms provided by the omnibus survey in Britain, the Health Survey for England and the Oxford Healthy Life Survey. J Public Health Med 1999:21:255-70.

18 Tabachnick BG, Fidell LS. Using multivariate statistics, 3rd ed. New York: Harper Collins, 1996.

19 Patten SB, Metz LM. Depression in multiple sclerosis. Psychother Psychosom 1997:66:286-92.

20 Feinstein A, Feinstein K. Depression associated with multiple sclerosis looking beyond diagnosis to symptom expression. J Affect Disord 2001;66:193-8.

21 Nortvedt MW, Riise T, Myhr KM, et al. Performance of the SF-36, SF-12, and RAND-36 summary scales in a multiple sclerosis population. Med Care 2000;38:1022-8. 